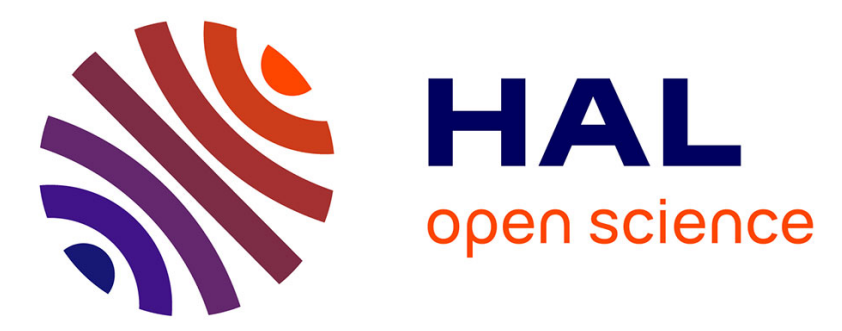

\title{
A study of the high temperature spin reorientation in YCoFe3B
}

F. Grandjean, M.-T. Sougrati, Hervé Mayot, Olivier Isnard, G. J. Long

\section{To cite this version:}

F. Grandjean, M.-T. Sougrati, Hervé Mayot, Olivier Isnard, G. J. Long. A study of the high temperature spin reorientation in YCoFe3B. Journal of Physics: Condensed Matter, 2009, 21, pp.186001. 10.1088/0953-8984/21/18/186001 . hal-00978114

\section{HAL Id: hal-00978114 \\ https://hal.science/hal-00978114}

Submitted on 26 Apr 2014

HAL is a multi-disciplinary open access archive for the deposit and dissemination of scientific research documents, whether they are published or not. The documents may come from teaching and research institutions in France or abroad, or from public or private research centers.
L'archive ouverte pluridisciplinaire HAL, est destinée au dépôt et à la diffusion de documents scientifiques de niveau recherche, publiés ou non, émanant des établissements d'enseignement et de recherche français ou étrangers, des laboratoires publics ou privés. 


\title{
A Study of the Unusual High Temperature Spin Reorientation in $\mathrm{YCoFe}_{3} \mathrm{~B}$
}

\section{Fernande Grandjean, ${ }^{1}$ Moulay T Sougrati, ${ }^{1}$ Hervé Mayot, ${ }^{2}$ Olivier Isnard, ${ }^{2}$ and Gary $\mathbf{J}$ Long $^{3}$}

\author{
${ }^{1}$ Department of Physics, B5, University of Liège, B-4000 Sart-Tilman, Belgium \\ ${ }^{2}$ Institut Néel, CNRS and Université de Grenoble Joseph Fourier, Avenue des Martyrs, BP166, F- \\ 38000 Grenoble, France \\ ${ }^{3}$ Department of Chemistry, Missouri University of Science and Technology, University of Missouri, \\ Rolla, Missouri 65409-0010, USA
}

E-mail: fgrandjean@ulg.ac.be, olivier.isnard@grenoble.cnrs.fr, glong@mst.edu

Received

\begin{abstract}
The iron-57 Mössbauer spectra of $\mathrm{YCoFe}_{3} \mathrm{~B}$ have been measured between 4.2 and $480 \mathrm{~K}$ and reveal that $\mathrm{YCoFe}_{3} \mathrm{~B}$ exhibits an axial orientation of the iron magnetic moments below $450 \mathrm{~K}$ and a basal orientation above $450 \mathrm{~K}$. This spin reorientation, also observed in the thermomagnetic curves, results from the different signs of the contributions to the magnetic anisotropy of the $2 c$ and $6 i$ sites that are occupied by iron. The neutron diffraction patterns of $\mathrm{YCoFe}_{3} \mathrm{~B}$ have been measured at $2 \mathrm{~K}$ and between 290 and $770 \mathrm{~K}$ and have been successfully analysed with a model compatible with the magnetic orientation obtained from the Mössbauer spectra. The hybridization between the cobalt or iron $3 d$ orbitals and the boron $2 p$ orbitals leads to a larger magnetic moment and hyperfine field on the $2 c$ site as compared to the $6 i$ site.
\end{abstract}

75.30.-m Intrinsic properties of magnetically ordered materials

76.80.+y Mössbauer effect

75.25.+z Spin arrangements in magnetically ordered materials (including neutron and spinpolarized electron studies, synchrotron-source x-ray scattering, etc.)

75.30.Gw Magnetic anisotropy

75.60.-d Domain effects, magnetisation curves, and hysteresis 


\section{Introduction}

The $R \mathrm{Co}_{4-x} \mathrm{Fe}_{x} \mathrm{~B}$ compounds, where $R$ is yttrium or a rare earth, have been extensively studied both because their crystalline structure derives from the technologically important $R \mathrm{Co}_{5}$ structures and because their magnetic properties and, more specifically, their magnetic anisotropy, strongly depend upon the iron content, $x$ [1-13].

Previous studies [8,9] of the $\mathrm{YCo}_{4-x} \mathrm{Fe}_{x} \mathrm{~B}$ compounds, where $x$ is 1,2 , and 3, reported that they crystallize in the hexagonal P6/mmn space group, exhibit an anisotropic expansion of the unit-cell, and show an increase in both their Curie temperatures and their $5 \mathrm{~K}$ saturation magnetisations with increasing $x$. The neutron diffraction [9,11] and Mössbauer spectral [12] studies indicate that the iron substitutes preferentially on the $2 c$ site. Finally, studies by powder neutron diffraction and $\mathrm{x}-$ ray diffraction on oriented powders indicate that the transition metal magnetic moments are oriented in the basal plane of the unit cell and do not exhibit [8,11] any spin reorientation between 4.2 and $295 \mathrm{~K}$ for $\mathrm{YCo}_{4-x} \mathrm{Fe}_{x} \mathrm{~B}$ when $x$ is 1 , 2, and 3. In contrast, $\mathrm{YCo}_{4} \mathrm{~B}$ exhibits a spin reorientation $[9,11,14]$ from basal below $100 \mathrm{~K}$ to axial above $150 \mathrm{~K}$, a spin reorientation that has been extensively studied as a function of temperature, applied field, and pressure on both polycrystalline and single crystal samples [15]. Finally, both an earlier Mössbauer spectral study [12] and neutron and x-ray diffraction measurements $[8,11]$ on an oriented powder of $\mathrm{YCoFe}_{3} \mathrm{~B}$ gave somewhat conflicting results for the orientation of the easy magnetisation axis below $295 \mathrm{~K}$. In addition, an anomaly [9] in the temperature dependence of the magnetisation of $\mathrm{YCO}_{4} \mathrm{~B}$ has been observed at ca. $450 \mathrm{~K}$.

In this paper, additional Mössbauer spectra between 4.2 and $480 \mathrm{~K}$ and neutron diffraction data between 2 and $770 \mathrm{~K}$ have been obtained and analyzed in detail together with the magnetisation data with the goal of clarifying the magnetic anisotropy of the iron magnetic moments.

\section{Experimental}

$\mathrm{YCoFe}_{3} \mathrm{~B}$ has been prepared as described earlier [8,9,11]. In order to reduce the absorption of thermal neutrons by the sample, isotopic boron-11 has been used rather that natural boron.

The Mössbauer spectra have been measured between 4.2 and $480 \mathrm{~K}$ on a constant-acceleration spectrometer which utilized a rhodium matrix cobalt-57 source and was calibrated at room temperature with $\alpha$-iron powder. The low temperature Mössbauer spectra were obtained in a Janis Supervaritemp cryostat and the absorber contained $25 \mathrm{mg} / \mathrm{cm}^{2}$ of powdered sample which had been sieved to a $0.045 \mathrm{~mm}$ or smaller diameter particle size. The high temperature Mössbauer spectra were obtained in a laboratory built vacuum oven and the absorber was a pressed pellet of a mixture of boron nitride and $40 \mathrm{mg} / \mathrm{cm}^{2}$ of $\mathrm{YCoFe}_{3} \mathrm{~B}$.

In order to determine the easy magnetisation direction, x-ray diffraction patterns have been obtained in reflection mode on powder samples oriented in an external magnetic field. Fieldoriented samples were prepared at room temperature by solidifying a mixture of epoxy resin and $\mathrm{YCoFe}_{3} \mathrm{~B}$ powder with grain size smaller than $50 \mu \mathrm{m}$ in a magnetic field of ca. $0.5 \mathrm{~T}$; the field was perpendicular to the plane of the sample. 
The Curie temperature was measured using a Faraday-type balance operating between 300 and $1000 \mathrm{~K}$ with a heating and cooling rate of $5 \mathrm{~K}$ per minute. The sample was sealed in an evacuated silica tube to avoid oxidation upon heating.

The neutron diffraction investigations were performed at the Institut Laue Langevin in Grenoble, France. The thermal dependence of the neutron diffraction patterns has been recorded at medium resolution on $\mathrm{D} 1 \mathrm{~B}$. The diffraction patterns were obtained over a $2 \theta$ angular range of $80^{\circ}$ by using a ${ }^{3} \mathrm{He}$ multidetector with a step of $0.2^{\circ}$ between each of the 400 detection cells. In its high flux neutron configuration mode used herein, D1B operates at a wavelength, $\lambda$, of $2.52 \AA$. A detailed description of this instrument may be found elsewhere [16].

A sample of $\mathrm{YCoFe}_{3} \mathrm{~B}$ powder was placed in a $6 \mathrm{~mm}$ diameter cylindrical vanadium sample holder. In order to detect and characterize any magnetic spin reorientation, several diffraction patterns have been obtained between 2 and $300 \mathrm{~K}$ in a cryostat and between 300 and $770 \mathrm{~K}$ in a furnace. The neutron powder diffraction data were analyzed by the Rietveld method with the Fullprof suite of programs [17]. Because the sample was prepared with boron-11, the neutron absorption originates mostly from the cobalt atoms and has been taken into account as previously described [18,19]. These calculated neutron diffraction absorption corrections were used in the Rietveld refinements under the assumption that the density of the sample in the sample holder was only $50 \%$ of the bulk density.

\section{X-ray Diffraction on Oriented Powders}

The room temperature $\mathrm{x}$-ray powder diffraction pattern obtained, in reflection geometry, on a powder of $\mathrm{YCoFe}_{3} \mathrm{~B}$ oriented under a magnetic field perpendicular to the plate of the sample is compared in Figure 1 with the calculated pattern for a random powder. The magnetic field reduces the intensity of many of the Bragg reflections including the most intense (103) and next most intense (102) reflections and enhances the intensity of the (003) and (004) reflections. These changes in intensity indicate that the magnetic moments in $\mathrm{YCoFe}_{3} \mathrm{~B}$ are oriented along the $c$-axis of its hexagonal unit cell at room temperature.

\section{Thermomagnetisation Curves}

The temperature dependence of the magnetisation of $\mathrm{YCoFe}_{3} \mathrm{~B}$ is shown in Figure 2 together, for comparison, with that of $\mathrm{YCo}_{3} \mathrm{FeB}$. A rather broad anomaly in the magnetisation is observed between 430 and $570 \mathrm{~K}$ in $\mathrm{YCoFe}_{3} \mathrm{~B}$, an anomaly that is not observed in either $\mathrm{YCo}_{3} \mathrm{FeB}$, see Figure 2, nor in $\mathrm{YCo}_{2} \mathrm{Fe}_{2} \mathrm{~B}$. However, a similar broad anomaly has been observed at spinreorientations in other intermetallic compounds that exhibit a competition between the magnetocrystalline anisotropy of different transition metals on their respective sublattices. [20-22] We have also attempted to determine the magnetic structure of $\mathrm{YCoFe}_{3} \mathrm{~B}$ on each side of the anomaly at ca. 410 and $590 \mathrm{~K}$. However, both because of the small magnetic contribution to the neutron scattering in $\mathrm{YCoFe}_{3} \mathrm{~B}$ and because of the relative proximity in temperature of the spinreorientation and Curie temperatures, no definitive conclusion concerning the existence of a spin reorientation or the changes in the magnetic structure could be obtained. Hence, a Mössbauer 
spectral study between 295 and $480 \mathrm{~K}$ has been undertaken because the Mössbauer spectra should be very sensitive to the orientation of the iron magnetic moments, [13] see the following discussion. The analysis of the neutron diffraction patterns obtained above $295 \mathrm{~K}$ will be discussed below in terms of the results obtained from the Mössbauer spectral study.

\section{Mössbauer Spectral Results}

The Mössbauer spectra of $\mathrm{YCoFe}_{3} \mathrm{~B}$ obtained from 4.2 to $85 \mathrm{~K}$ are shown in Figure 3 and the spectra obtained from 300 to $480 \mathrm{~K}$ are shown in Figure 4. First, it is apparent that the low temperature spectra are similar to the 78 and $295 \mathrm{~K}$ spectra previously measured [12] on a different absorber and on a different spectrometer, spectra that are characteristic $[12,13]$ of an axial orientation of the iron magnetic moments. Second, it is apparent that above $400 \mathrm{~K}$, in addition to the expected decrease in hyperfine field with increasing temperature, the Mössbauer spectral profile changes significantly, a change [13] that indicates the presence of a spin reorientation.

Because of the simultaneous presence of both small hyperfine fields and large quadrupole interactions, a simulation of the iron-57 Mössbauer spectra of $\mathrm{YCoFe}_{3} \mathrm{~B}$ is not possible within the first-order perturbation approximation and requires an exact solution [12]. The resulting fits are shown as the black lines in Figures 3 and 4 where the blue lines correspond to the $2 c$ sites and the red lines correspond to the $6 i$ sites; the corresponding site averaged isomer shifts, $\delta$, quadrupole interactions, $\Delta E_{Q}$, hyperfine fields, $H$, and relative areas, and their statistical errors, are given in Table 1. The absolute errors associated with these parameters are approximately twice as large as the statistical errors. In addition to the dominant $\mathrm{YCoFe}_{3} \mathrm{~B}$ phase, iron-containing impurities are present and are most apparent at ca. 5 and $6 \mathrm{~mm} / \mathrm{s}$ in the low temperature spectra. These impurities have been fit with two sextets whose total relative area amounts to ca. 7 percent of the observed spectral area and whose hyperfine parameters are compatible with the presence of an FeCo alloy. No further reference to these impurities will be made in the following discussion.

The specific details concerning the fits and the constrains imposed upon the hyperfine parameters are the same as those used earlier in the analysis of the spectra of the $\mathrm{YCo}_{4-} \mathrm{Fe}_{x} \mathrm{~B}$ and $\mathrm{GdCo}_{4-\mathrm{x}} \mathrm{Fe}_{x} \mathrm{~B}$ compounds $[12,13]$. The asymmetry parameter, $\eta$, of the $2 c$ and $6 i$ sites has been constrained to 0 and 1, respectively, in agreement with the point symmetry of these sites [6]. For an axial orientation of the iron magnetic moment and, hence, the hyperfine field, the Euler angles, $\theta$ and $\phi$, of the hyperfine field in the electric field gradient axes are $0^{\circ}$ and $0^{\circ}$ for the $2 c$ site and $90^{\circ}$ and $0^{\circ}$ for the $6 i$ site. The Euler angles have been constrained to these values for all the fits of the spectra obtained at and below $400 \mathrm{~K}$. In contrast, for a basal orientation of the iron magnetic moments, the Euler angles, $\theta$ and $\phi$, for the $2 c$ site are $90^{\circ}$ and $0^{\circ}$, respectively. Further, because the principal axis of the electric field gradient, $V_{z z}$, of the $6 i$ site is along [100], the $6 i$ site must be subdivided into two magnetically inequivalent sites, herein designated as the $6 i_{1}$ and $6 i_{2}$ subsites, with relative populations of one and two, respectively. In this case, the angles, $\theta$ and $\phi$, of the hyperfine field are $0^{\circ}$ and $90^{\circ}$ for the $6 i_{1}$ subsite and $120^{\circ}$ and $90^{\circ}$ for the $6 i_{2}$ subsite, respectively, and the Euler angles have been constrained to these values for the fits of the spectra obtained at 450 
and $480 \mathrm{~K}$. The line widths, isomer shifts, and quadrupole interactions of the $6 i_{1}$ and $6 i_{2}$ subsites have been constrained to be the same at all temperatures.

In addition to adjusting the isomer shift, quadrupole splitting, and hyperfine field, for both the $2 c$ and $6 i$ sites in $\mathrm{YCoFe}_{3} \mathrm{~B}$, and their line width, an incremental line width has also been fit for the $6 i$ site in order to account for the distribution of cobalt and iron on its near-neighbours. This unitless incremental line width, $\Delta \Gamma$, has been defined as $\Gamma(v)=\Gamma+(v-\delta) \Delta \Gamma$, where $v$ is the velocity and $\delta$ is the isomer shift of a given spectral component. The relative areas of the $2 c$ and $6 i$ components have been adjusted starting from the initial values given by the iron occupancies obtained from the neutron powder diffraction results, but as noted previously [12], the $2 c$ contribution to the Mössbauer spectral area was underestimated. Hence, the relative areas have been fit and are given in Table 1.

In order to account for the spectral profile and, more specifically, for the line profile observed at $-5 \mathrm{~mm} / \mathrm{s}$ in the spectra obtained at $400 \mathrm{~K}$ and below, it was necessary to include four subcomponents for the $2 c$ site and two subcomponents for the $6 i$ site. These components reflect the change in hyperfine parameters with the distribution of cobalt and iron on the $2 c$ and $6 i$ sites. At 450 and $480 \mathrm{~K}$, the decrease in spectral resolution, a decrease that is a normal consequence of the decrease in the hyperfine field and in recoil-free fraction, only two broadened $6 i_{1}$ and $6 i_{2}$ components and one $2 c$ component are required to fit these spectra. The relative area of ca. $33 \%$ observed for the $2 c$ contribution to the Mössbauer spectra over the entire temperature range corresponds to a full occupation of the $2 c$ site by iron in good agreement with the neutron diffraction results $[9,11]$.

The temperature dependence of the $2 c$ and $6 i$ site weighted average isomer shift in $\mathrm{YCoFe}_{3} \mathrm{~B}$ is shown in Figure 5. The red solid line results from a fit with the second-order Doppler shift with a Mössbauer temperature of $447(75) \mathrm{K}$ for the $6 i$ site. Above $300 \mathrm{~K}$, the $2 c$ isomer shift is more negative than the $6 i$ isomer shift. There is no systematic trend $[6,12,13,23]$ in the relative values of the isomer shift of the $2 c$ and $6 i$ sites in the $R \mathrm{Co}_{4-x} \mathrm{Fe}_{x} \mathrm{~B}$, where $R$ is $\mathrm{Sm}, \mathrm{Gd}, \mathrm{Dy}$, and $\mathrm{Tb}$ and $x$ is 1 to 3 . The quadrupole interactions are virtually independent of temperature.

The temperature dependence of the $2 c$ and $6 i$ site weighted average hyperfine field in $\mathrm{YCoFe}_{3} \mathrm{~B}$ is shown in Figure 6a. The $2 c$ hyperfine field is larger than the $6 i$ hyperfine field for both the axial and basal orientations of the iron magnetic moments. The same relative values of the hyperfine fields have been observed [12,13,23] in the $R \mathrm{Co}_{4-x} \mathrm{Fe}_{x} \mathrm{~B}$, where $R$ is $\mathrm{Gd}$, Dy, and $\mathrm{Tb}$ and $x$ is 1 to 3, and agree with the larger magnetic moment measured $[9,11]$ on the $2 c$ site by neutron diffraction. This general trend reflects the difference in local environment of the two inequivalent iron sites, a trend that agrees with an earlier study [24]. The volume, the local symmetry of the iron WignerSeitz cell as well as the iron-metalloid bonding are important factors in determining the magnitude of the iron magnetic moments in intermetallic compounds.

A plot of the reduced hyperfine field versus reduced temperature for the $2 c$ and $6 i$ sites is shown in Figure 6b. In this plot the Curie temperature is taken equal to $673 \mathrm{~K}$ and the saturation hyperfine field for $2 c$ and $6 i$ sites are taken equal to 24 and $19 \mathrm{~T}$, respectively. The black solid line is a Brillouin curve for a spin 5/2. It is clear that the reduced $6 i$ hyperfine field follows well the 
Brillouin curve up to the spin reorientation, a reorientation that occurs at a reduced temperature of ca. 0.63 . The $2 c$ reduced hyperfine field shows a somewhat different dependence.

\section{Neutron Diffraction Results}

A Rietveld refinement of the 2 and $300 \mathrm{~K}$ neutron diffraction patterns of $\mathrm{YCoFe}_{3} \mathrm{~B}$ has been carried out on the basis of the orientation of the iron magnetic moments obtained from the above Mössbauer spectral study. The $2 \mathrm{~K}$ refinement is shown in Figure 7 and the resulting parameters are given in Table 2. Hence, the axial orientation of the iron magnetic moment revealed by the Mössbauer spectra is also compatible with the weak magnetic scattering in the neutron diffraction patterns obtained at 2 and $300 \mathrm{~K}$. Indeed, the total magnetic contribution to the diffracted intensity corresponds to only 6 percent of the total scattering.

The mean Fe-Co magnetic moment obtained from the $2 \mathrm{~K}$ refinement of the two inequivalent transition metal sites in $\mathrm{YCoFe}_{3} \mathrm{~B}$ are 2.1(2) and 1.0(1) $\mu_{\mathrm{B}}$ for the $2 c$ and $6 i$ sites, respectively. The smaller moment observed for the $6 i$ site confirms the large influence of the hybridization of the $6 i$ $\mathrm{Co}(\mathrm{Fe}) 3 d$ orbitals with the boron $2 p$ orbitals. Refinement of the $300 \mathrm{~K}$ pattern led to similar values of the mean Fe-Co magnetic moment as would be expected at a temperature that is well below the Curie temperature of $673 \mathrm{~K}$.

A comparison of the diffraction patterns of $\mathrm{YCoFe}_{3} \mathrm{~B}$, obtained below and above the spinreorientation, at 373 and $573 \mathrm{~K}$ is shown in Figure 8. It is obvious that these two very similar patterns cannot provide a definitive indication of the presence of a spin-reorientation between 373 and $573 \mathrm{~K}$. The main difference between these two patterns is a small intensity reduction of the low angle (100) Bragg reflection upon increasing temperature, a reduction that is expected as the temperature approaches the Curie temperature. Other patterns have been obtained between 300 and $770 \mathrm{~K}$ and further support the conclusion that, because of the weak magnetic scattering contribution to the neutron diffraction pattern, no definitive conclusion can be reached on the direction of the magnetic moments based solely on neutron diffraction studies. However, these patterns can be satisfactorily analyzed on the basis of the direction of the iron magnetic moments obtained from the Mössbauer spectra obtained above and below the spin-reorientation.

\section{Discussion and Conclusions}

The Mössbauer spectra obtained at 400 and $450 \mathrm{~K}$ conclusively prove that a spin reorientation occurs in $\mathrm{YCoFe}_{3} \mathrm{~B}$ between these temperatures, a spin reorientation that is also indicated by the broad anomaly in the temperature dependence of the magnetisation observed between 430 and 570 $\mathrm{K}$, see Figure 2. This spin reorientation has been predicted [12] from an individual site magnetic anisotropy model $[25,26]$, in which the first-order magnetic anisotropy constant, $K_{1}$, is given by

$$
K_{1}=K_{1}(0)+2 f_{2 c} \Delta K_{1}{ }^{2 c}+6 f_{6 i} \Delta K_{1}^{6 i},
$$

where $K_{1}(0)=5 \times 10^{5} \mathrm{~J} / \mathrm{m}^{3}$ is the magnetic anisotropy constant for $x=0, f_{2 c}$ and $f_{6 i}$ are the iron occupancies, and $\Delta K_{1}{ }^{2 c}=-7.55 \times 10^{5} \mathrm{~J} / \mathrm{m}^{3}$ and $\Delta K_{1}^{6 i}=+3.55 \times 10^{5} \mathrm{~J} / \mathrm{m}^{3}$ are the differences in the iron and cobalt contributions to the magnetic anisotropy for the $2 c$ and $6 i$ sites, respectively. The spin reorientation occurs for $x=3$ in the $\mathrm{YCo}_{4-x} \mathrm{Fe}_{x} \mathrm{~B}$ compounds, because the $6 i$ sites are 
approximately half occupied by iron and the positive contribution of the $6 i$ site to the magnetic anisotropy dominates the negative contribution of the $2 c$ site.

$\mathrm{YCo}_{4} \mathrm{~B}$ is known to exhibit a spin-reorientation transition as a result of the competition between the anisotropy of the two inequivalent cobalt crystallographic sites. Further, iron and cobalt are known to exhibit an opposite sign for the magnetocrystalline anisotropy in isotypic compounds. Upon the substitution of iron for cobalt in the $\mathrm{YCo}_{4} \mathrm{~B}$ structure, the iron preferentially occupies the $2 c$ site thus reducing the uniaxial anisotropic magnetic character of the compound and, consequently $\mathrm{YCo}_{3} \mathrm{FeB}$ and $\mathrm{YCo}_{2} \mathrm{Fe}_{2} \mathrm{~B}$ exhibit planar anisotropy over the entire range of ordered temperatures. In contrast, in $\mathrm{YCoFe}_{3} \mathrm{~B}$ the larger iron content leads to a significant occupation of the $6 i$ site by iron and to a competition between the $\mathrm{Fe}_{2 c}$ and $\mathrm{Fe}_{6 i}$ contributions to the magnetocrystalline anisotropy, a competition that leads to the appearance of a spin reorientation between 400 and $450 \mathrm{~K}$. Again, in contrast to $\mathrm{YCo}_{4} \mathrm{~B}$, whose easy magnetization direction lies in the basal hexagonal plane at low temperature and along the $c$-axis above the spin reorientation $[9,11,14], \mathrm{YCoFe}_{3} \mathrm{~B}$ exhibits uniaxial alignment of the magnetic moment below its spin reorientation and a basal anisotropy at temperatures above its spin reorientation. This observation confirms the opposite character of the anisotropy of iron and cobalt in the $\mathrm{YCo}_{4-x} \mathrm{Fe}_{x} \mathrm{~B}$ compounds.

Both the hyperfine fields obtained from the Mössbauer spectra and the magnetic moments obtained from the neutron diffraction patterns demonstrate the sensitivity of the magnitude of the iron magnetic moment to the presence of boron in its environment.

\section{Acknowledgments.}

The authors thank Dr. Raphaël P Hermann for his help in developing the code to analyze the Mössbauer spectra and to Dr. C Chacon for interesting discussions at the start of this work. This work was partially supported by the Fonds National de la Recherche Scientifique, Belgium, through grants 9.456595 and 1.5.064.05, by the "Commissariat Général aux Relations Internationales, Ministère de la Communauté Française de Belgique, Relations scientifiques avec la France," grant 2007/02242/S, and by the CNRS-cooperation program No. PVB/ADK/FR/0084-22/03/2006-091-S. 


\section{References}

[1] Kuz'ma Y B and Bilonizhko N S 1974 Soviet Phys.-Crystallogr. 18447

[2] Kuz'ma Y B, Bilonizhko N S, Mykhalenko S I, Stepanova G F and Chaban N F 1979 J. LessCommon Met. 6751

[3] El-Masry N A and Stadelmaier H H 1983 Z. Metallkunde 7486

[4] Spada F, Abache C and Oesterreicher H 1984 J. Less-Common Met. 99 L21

[5] Jiang S Y, Wallace W E and Burzo E 1986 J. Magn. Magn. Mater. 61257

[6] Gros Y, Hartmann-Boutron F, Meyer C, Fremy M A and Tenaud P 1988 J. Magn. Magn. Mater. 74319

[7] Drzazga Z, Popiel E and Winiarska A 1992 J. Magn. Magn. Mater. 104-107 1437

[8] Chacon C and Isnard O 2000 Physica B 276-278 652

[9] Chacon C 2000 Doctoral Dissertation, Université Joseph Fourier - Grenoble I

[10] Maruyama F, Amako Y and Nagai H 2007 J. Alloys Compounds 42972

[11] Chacon C and Isnard O 2001 J. Appl. Phys. 8971

[12] Long G J, Hermann R P, Grandjean F, Chacon C and Isnard O 2006 J. Phys.: Cond. Matter 18 10765

[13] Grandjean F, Hermann R P, Popiel E and Long G J 2007 J. Appl. Phys. 101023917

[14] Thang C V, Brommer P E, Thuy N P and Franse J J M 1997 J. Magn. Magn. Mater. 171237

[15] Mayot H, Isnard O, Arnold Z and Kamarad J 2008 J. Phys.: Condens. Matter 20135207

[16] www.ill.eu/d1b/

[17] Rodriguez Carjaval J 1993 Physica B 19255

[18] Sears V F 1992 Neutron News 326

[19] Hewat A W 1979 Acta Cryst. A35 248

[20] Isnard O, Soubeyroux J L, Fruchart D, Jacobs T H and Buschow K H J 1992 J. Phys.: Condens. Matter 46367

[21] LeCaer G, Malaman B, Isnard O, Soubeyroux J L, Fruchart D, Jacobs T H and Buschow K H J 1993 Hyperfine Interactions 77221

[22] Isnard O, Arnold Z, Kamarad J and Buschow K H J 2007 J. Appl. Phys. 101103908

[23] Mayot H, Isnard O, Grandjean F and Long G J 2008 J. Appl. Phys. 103093917

[24] Isnard O and Fruchart D 1994 J. Alloys Compounds 2051

[25] Thang C V, Thuy N P, Liu J P, Hien N T and Hien T D 1995 J. Magn. Magn. Mater. 14745

[26] Franse J J M, Kayzel F E and Thuy N P 1992 J. Magn. Magn. Mater. 1291211 
Table I. The Site Averaged Mössbauer Spectral Parameters for $\mathrm{YCoFe}_{3} \mathrm{~B} .{ }^{a}$

\begin{tabular}{|c|c|c|c|c|c|c|c|c|c|c|}
\hline \multirow[b]{2}{*}{$\begin{array}{l}\mathrm{T}, \\
\mathrm{K}\end{array}$} & \multicolumn{5}{|c|}{$2 c$} & \multicolumn{5}{|c|}{$6 i$} \\
\hline & $\begin{array}{l}\delta,^{\mathrm{b}} \\
\mathrm{mm} / \mathrm{s}\end{array}$ & $\begin{array}{l}\Delta E_{Q} \\
\mathrm{~mm} / \mathrm{s}\end{array}$ & $\begin{array}{l}H, \\
\mathrm{~T}\end{array}$ & $\begin{array}{l}\text { Area, } \\
\%\end{array}$ & $\Delta \Gamma$ & $\begin{array}{l}\delta,^{\mathrm{b}} \\
\mathrm{mm} / \mathrm{s}\end{array}$ & $\begin{array}{l}\Delta E_{Q} \\
\mathrm{~mm} / \mathrm{s}\end{array}$ & $\begin{array}{l}H, \\
\mathrm{~T}\end{array}$ & $\begin{array}{l}\text { Area, } \\
\%\end{array}$ & $\Delta \Gamma$ \\
\hline 480 & $-0.30(2)$ & -0.7 & $16.3(1)$ & $38(2)$ & 0 & $-0.16(5)$ & 0.9 & $11.5(2)$ & $62(2)$ & 0 \\
\hline 450 & $-0.25(2)$ & -0.7 & $16.7(1)$ & $31(2)$ & 0 & $-0.22(1)$ & 0.9 & $13.2(2)$ & $69(2)$ & 0 \\
\hline 400 & $-0.26(2)$ & $-0.87(4)$ & $18.8(1)$ & $31(2)$ & 0.05 & $-0.148(7)$ & $0.83(4)$ & $15.8(2)$ & $69(2)$ & 0.05 \\
\hline 350 & $-0.19(1)$ & $-1.10(4)$ & $20.3(1)$ & $31(2)$ & 0.05 & $-0.094(5)$ & $0.91(4)$ & $16.5(2)$ & $69(2)$ & 0.05 \\
\hline 300 & $-0.13(1)$ & $-1.08(2)$ & $21.31(5)$ & $31(2)$ & 0.05 & $-0.062(2)$ & $0.96(2)$ & $17.2(2)$ & $69(2)$ & 0.05 \\
\hline 85 & $0.047(5)$ & $-0.94(1)$ & $24.71(5)$ & $22(1)$ & 0 & $0.040(7)$ & $0.99(1)$ & $18.6(1)$ & $78(1)$ & 0.03 \\
\hline 60 & $0.059(5)$ & $-0.95(1)$ & $24.79(5)$ & $22(1)$ & 0 & $0.043(1)$ & $0.98(1)$ & $18.7(1)$ & $78(1)$ & 0.03 \\
\hline 40 & $0.054(5)$ & $-0.52(1)$ & $24.83(5)$ & $22(1)$ & 0 & $0.046(1)$ & $0.99(1)$ & $18.7(1)$ & $78(1)$ & 0.03 \\
\hline 20 & $0.036(5)$ & $-0.89(1)$ & $24.82(5)$ & $22(1)$ & 0 & $0.051(2)$ & $0.98(1)$ & $18.8(1)$ & $78(1)$ & 0.03 \\
\hline 4.2 & $0.029(5)$ & $-0.87(1)$ & $24.88(5)$ & $22(1)$ & 0 & $0.051(1)$ & $0.97(1)$ & $18.8(1)$ & $78(1)$ & 0.03 \\
\hline
\end{tabular}

${ }^{\mathrm{a}}$ The errors on the average are the statistical errors. More realistic errors may be twice as large. No error is given for the parameters that have been constrained to the value given. ${ }^{\mathrm{b}}$ The isomer shifts are given relative to room temperature $\alpha$-iron powder. 
Table 2. The $2 \mathrm{~K}$ Rietveld refinement and reliability parameters obtained from the powder neutron diffraction pattern of $\mathrm{YCoFe}_{3} \mathrm{~B}$.

\begin{tabular}{ll}
\hline \hline$z 6 i$ & $0.281(2)$ \\
$2 c$ iron occupancy, \% & $81.9(2)$ \\
$6 i$ iron occupancy, \% & $72.8(2)$ \\
$\mu_{2 c}, \mu_{\mathrm{B}}$ & $2.1(2)$ \\
$\mu_{6 i}, \mu_{\mathrm{B}}$ & $1.0(1)$ \\
$R_{w p}$ & 12.1 \\
$R_{\text {exp }}$ & 1.4 \\
$R_{\text {Bragg }}$ & 5.0 \\
$R_{\text {mag }}$ & 9.7 \\
\hline \hline
\end{tabular}




\section{Figure Captions}

Figure 1. The calculated $\mathrm{x}$-ray diffraction pattern for a random powder of $\mathrm{YCoFe}_{3} \mathrm{~B}$, bottom, and the experimental $\mathrm{x}$-ray diffraction pattern obtained on an oriented powder of $\mathrm{YCoFe}_{3} \mathrm{~B}$, top, with the magnetic easy-axis perpendicular to the plane of the sample. The * indicates peaks assigned to a trace of oxide impurity.

Figure 2. Thermomagnetisation curves obtained for the $\mathrm{YCo}_{4-x} \mathrm{Fe}_{x} \mathrm{~B}$ compounds with $x=1$ and 3 . The curve for $x=3$ has been increased by 3.58 .

Figure 3. The Mössbauer spectra of $\mathrm{YCoFe}_{3} \mathrm{~B}$ obtained at and below $85 \mathrm{~K}$. The black lines through the data points are the result of the fits discussed in the text. The very weak black sextets correspond to magnetic impurities and the blue and red lines correspond to the sum of the $2 c$ and $6 i$ components.

Figure 4. The Mössbauer spectra of $\mathrm{YCoFe}_{3} \mathrm{~B}$ obtained at $300 \mathrm{~K}$ and above. The black lines are the result of the fits discussed in the text and the blue and red lines correspond to the sum of the $2 c$ and $6 i$ components.

Figure 5. The temperature dependence of the site weighted average isomer shift obtained for the $2 c$ site, blue squares, and the $6 i$ site, red points, in $\mathrm{YCoFe}_{3} \mathrm{~B}$. The solid line corresponds to a fit with the second-order Doppler shift model for the $6 i$ site.

Figure 6. (a) The temperature dependence of the site weighted average hyperfine fields of the $2 c$ site, blue squares, and the $6 i$ site, red points, in $\mathrm{YCoFe}_{3} \mathrm{~B}$. (b) A reduced plot of the $2 c$ and $6 i$ hyperfine fields. The black solid line is a Brillouin curve for a spin $5 / 2$. The spin reorientation occurs at a reduced temperature of 0.63 in this plot.

Figure 7. The neutron powder diffraction pattern of $\mathrm{YCoFe}_{3} \mathrm{~B}$ obtained at $2 \mathrm{~K}$. The red points are the observed intensity and the solid line is the result of the fit described in the text, the vertical bars indicate the positions of the nuclear and magnetic diffraction reflections, and the bottom line is the difference between the experimental points and the calculated diffraction pattern.

Figure 8. The neutron powder diffraction pattern of $\mathrm{YCoFe}_{3} \mathrm{~B}$ obtained below the spinreorientation at $373 \mathrm{~K}$, bottom, and above the spin-reorientation at $573 \mathrm{~K}$, top. 

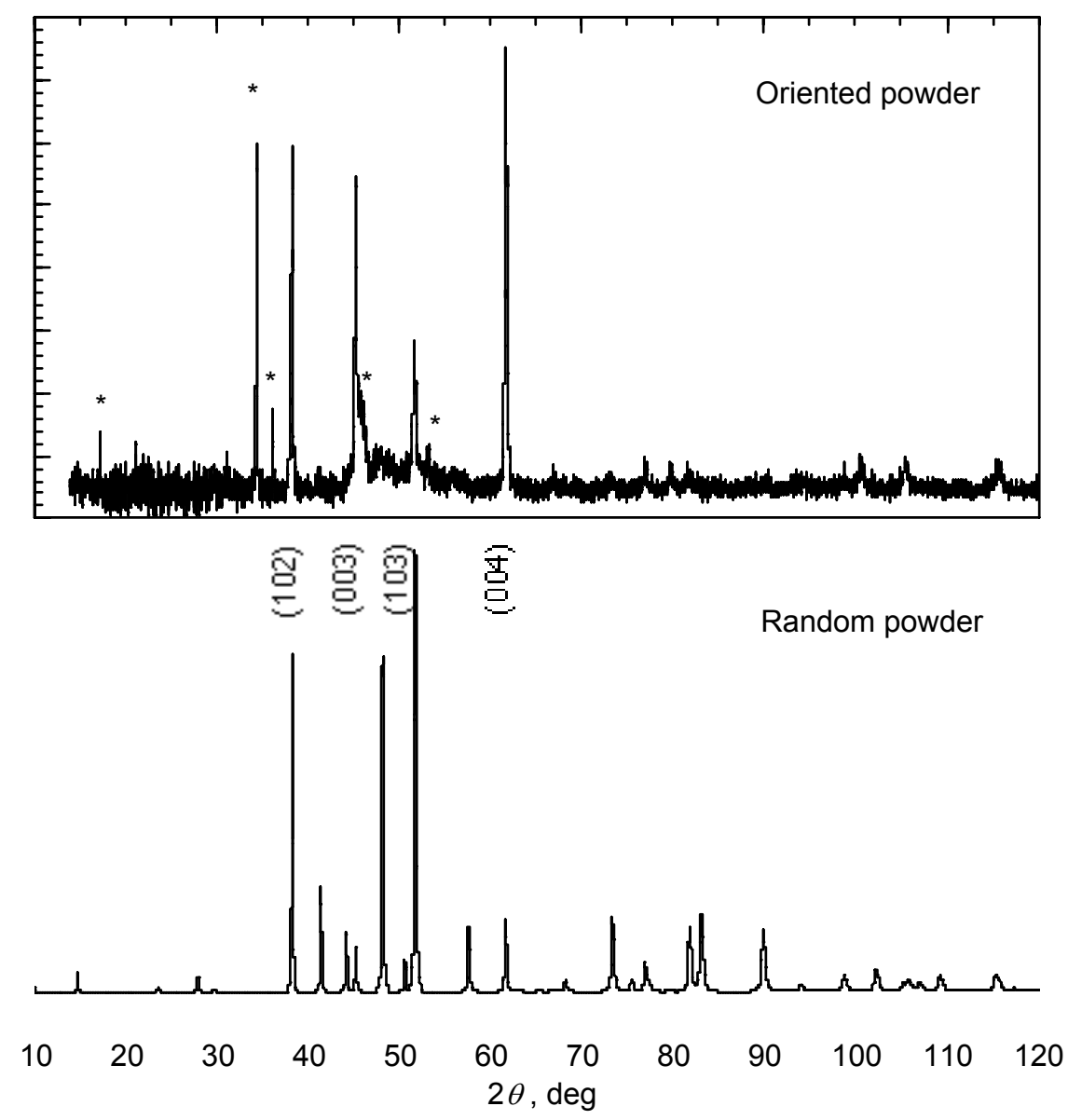

Figure 1. 


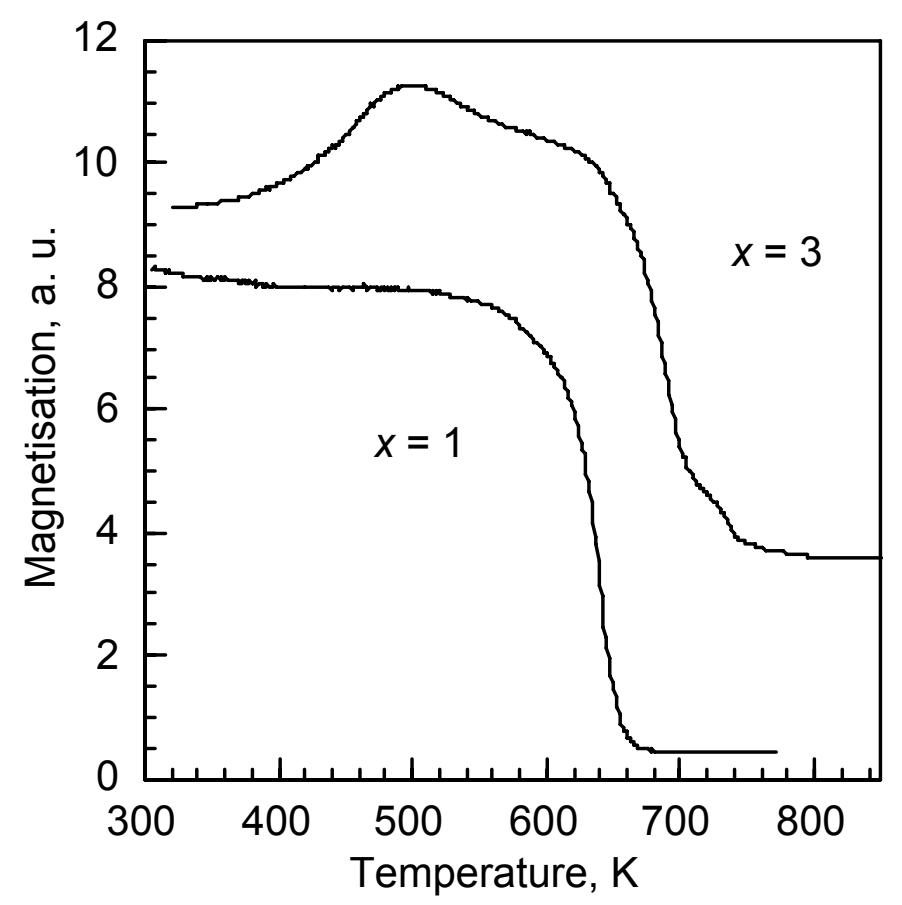

Figure 2. 
Figure 3. 


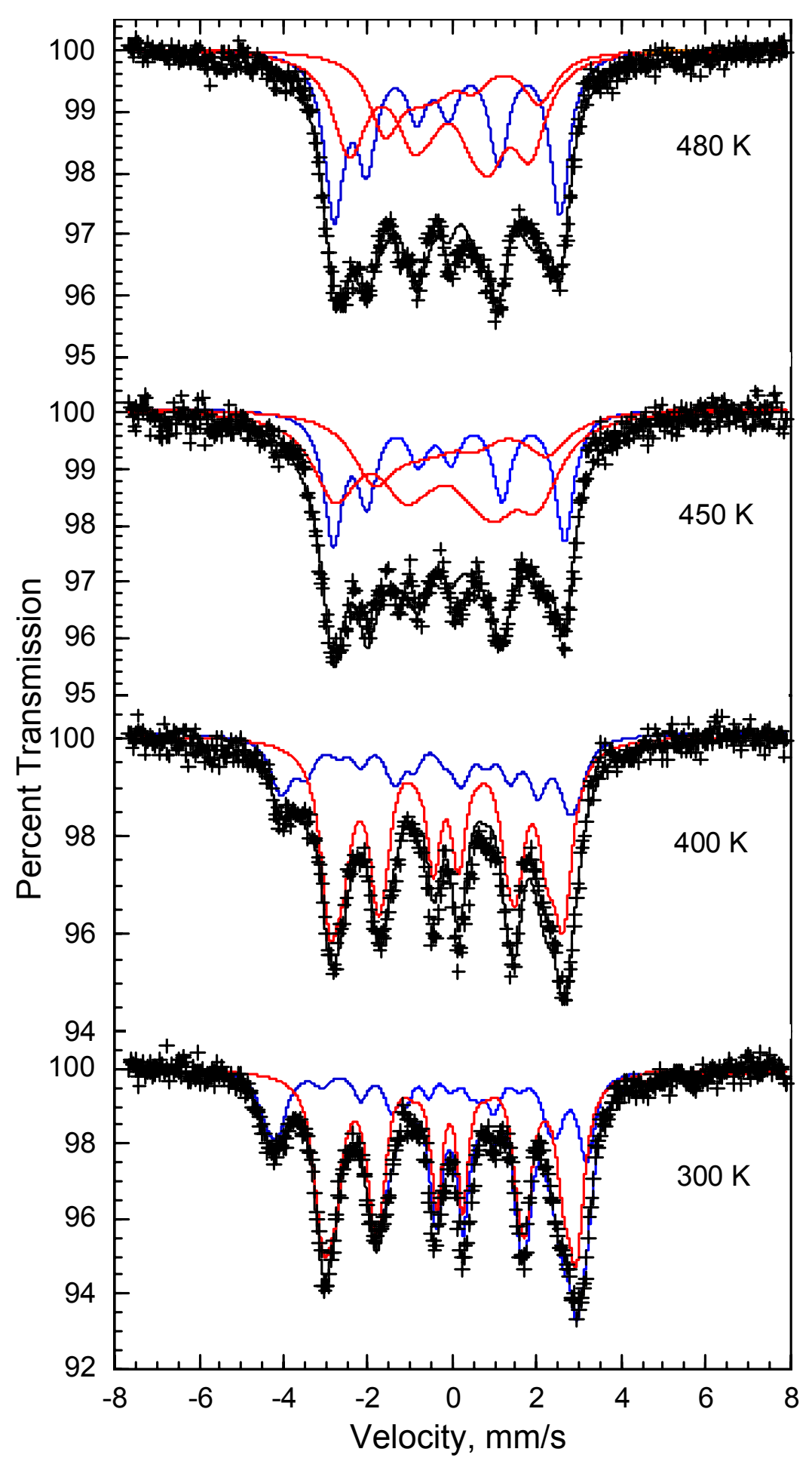

Figure 4. 


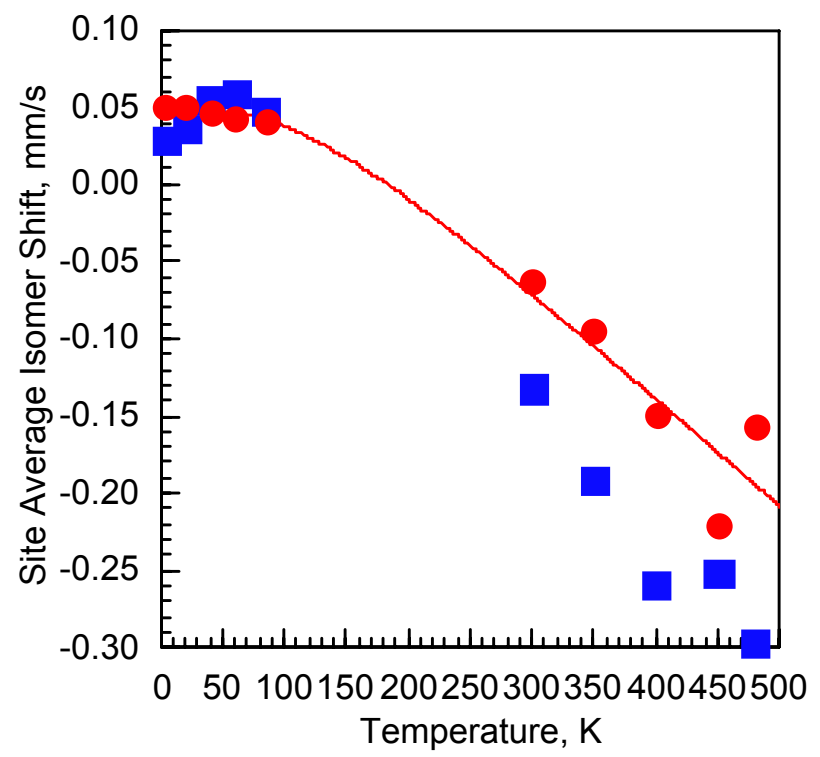

Figure 5. 

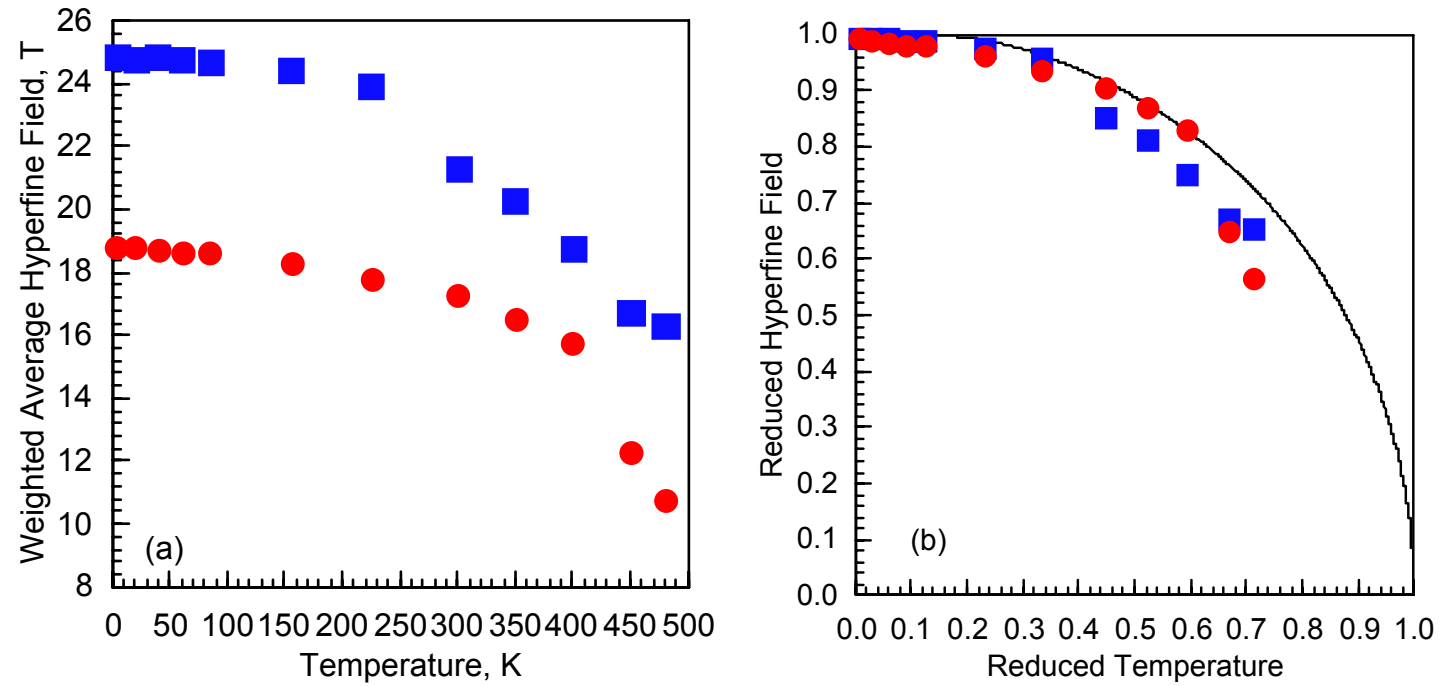

Figure 6. 


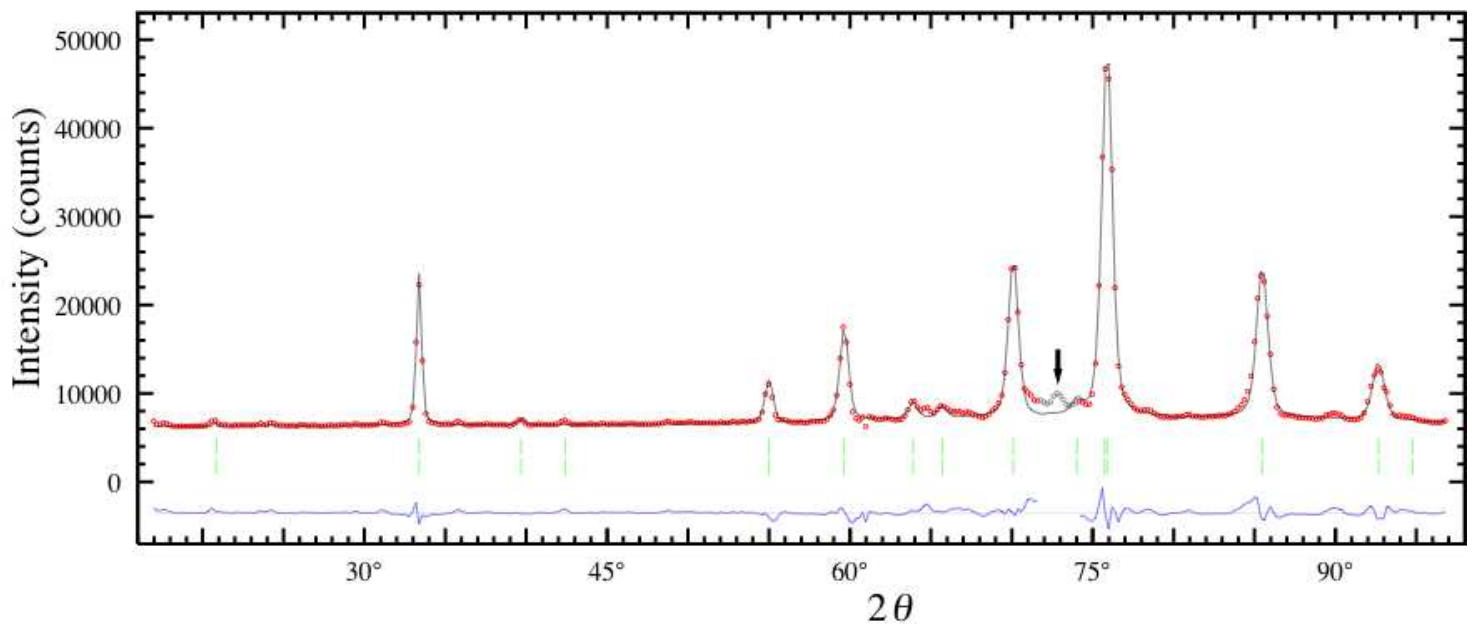

Figure 7. 


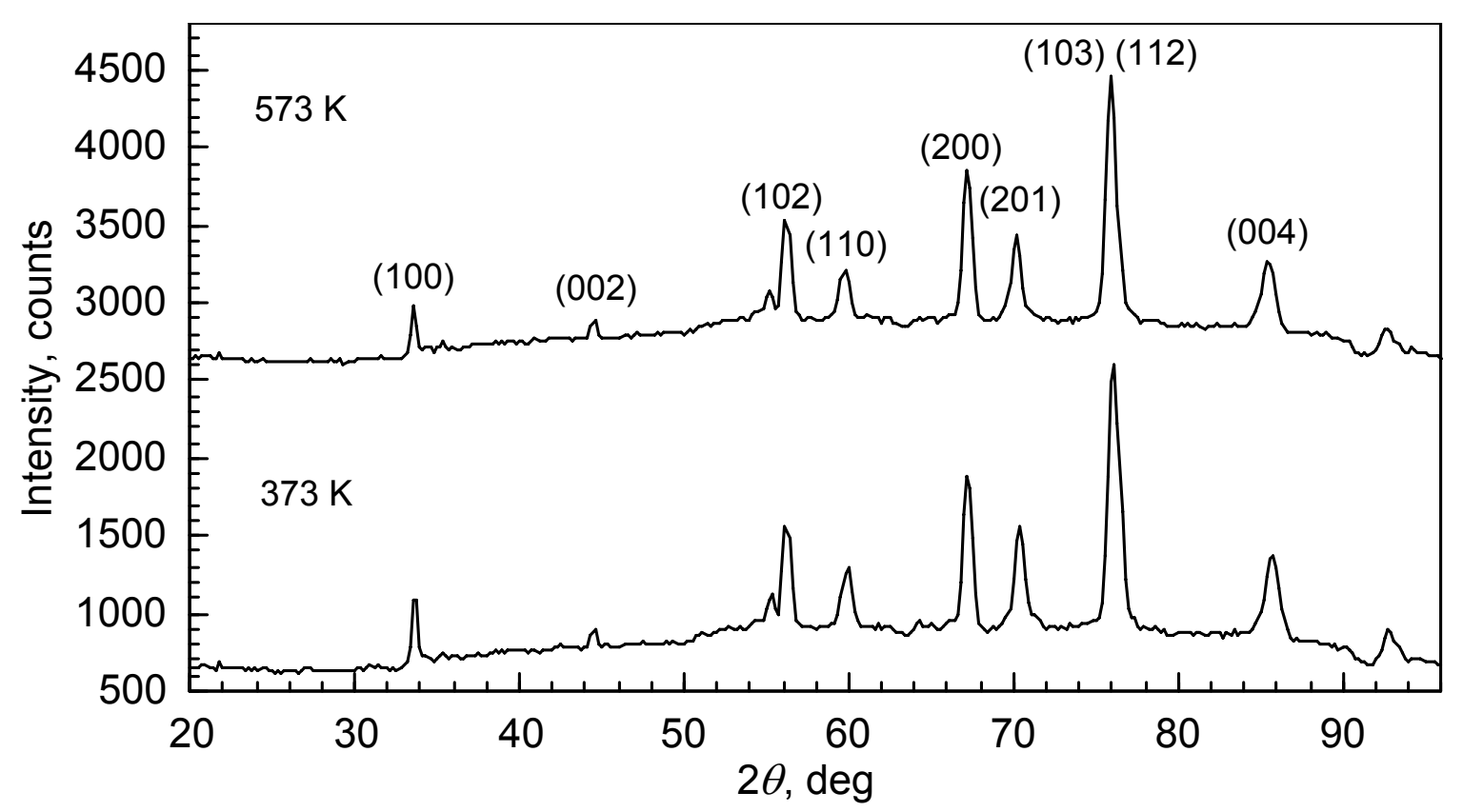

Figure 8. 